\title{
Threshold Behavior \\ for Nonlinear Wave Equations
}

\author{
Piotr BIZON \\ Institute of Physics, Jagellonian University, Kraków, Poland \\ E-mail: bizon@thrisc.if.uj.edu.pl
}

\begin{abstract}
In this brief contribution, which is based on my talk at the conference, I discuss the dynamics of solutions of nonlinear wave equations near the threshold of singularity formation. The heuristic picture of threshold behavior is first presented in a general setting and then illustrated with three examples.
\end{abstract}

Consider an initial value problem

$$
\frac{d u}{d t}=A(u), \quad u(0)=\phi \in \mathcal{D},
$$

where $A$ is a nonlinear operator. We can say that we understand qualitatively the dynamics of solutions of (1) if we can map the space of initial data $\mathcal{D}$ into a space of all possible final states (attractors). To construct such a mapping one needs first to find all generic (that is stable) attractors. If there are two or more stable attractors and the space $\mathcal{D}$ is connected, then there arises a problem of determining the boundaries between the basins of attraction of generic attractors. Here I discuss this problem in the case when (1) is a nonlinear wave equation whose solutions admit only two generic asymptotic behaviors, namely dispersion or blowup. By dispersion I mean that the solution exists globally in time and the energy density asymptotically decays to zero in any compact region. By blowup I mean that either the solution or some of its derivatives become unbounded in some norm (usually in finite time). A canonical example of equations I consider here is the nonlinear Klein-Gordon equation

$$
u_{t t}=\Delta u-u+u^{3}
$$

with the sign of the cubic term corresponding to the focusing effect of nonlinearity. For equations like this one expects that small initial data solutions exist globally, while large initial data solutions blow up in finite time. The basic idea behind this expectation is that for small initial data the nonlinear focusing term remains small for some time which is long enough for the spreading effect of Laplacian to take over. By contrast, for large initial data the singularizing effect of nonlinearity dominates and the solution blows up. In the following the boundary between global existence and blowup will be referred to as the threshold for singularity formation (or just threshold for brevity). The determination of the threshold and the dynamics near the threshold is of great interest in physical models which predict formation of singularities, for example in general relativity. Unfortunately, in most cases the problem seems to be beyond currently available rigorous techniques so it is indispensable to use mixed analytic and numerical methods (perhaps for this reason the

Copyright (c) 2001 by P Bizoń 
problem has received little attention from mathematicians). Below I sketch the general strategy of studying the threshold behavior and the underlying heuristic picture ${ }^{1}$.

The threshold can be located numerically as follows. Consider a one-parameter family of initial data $\phi(p)$ such that the corresponding solutions exist globally if the parameter $p$ is small or blow up if the parameter $p$ is large. Then, along the curve $\phi(p)$ there must exist a critical value $p^{*}$ which separates these two regimes. Given two values $p_{\text {small }}$ and $p_{\text {large }}$, it is straightforward (in principle) to find $p^{*}$ by bisection. Repeating this for many different interpolating families of initial data one obtains a set of critical data $\left\{\phi_{i}\left(p_{i}^{*}\right)\right\}$ which by construction belongs to the threshold. In order to figure out the structure of the threshold one needs to determine the flow of critical data. The precisely critical data cannot be prepared numerically but in practice it is sufficient to follow the evolution of marginally critical data. One finds that the flow of such data has a universal (that is familyindependent) transient phase when it seems to approach a particular "universal solution", a sort of intermediate attractor. This kind of behavior can be naturally explained as follows (see the figure below).

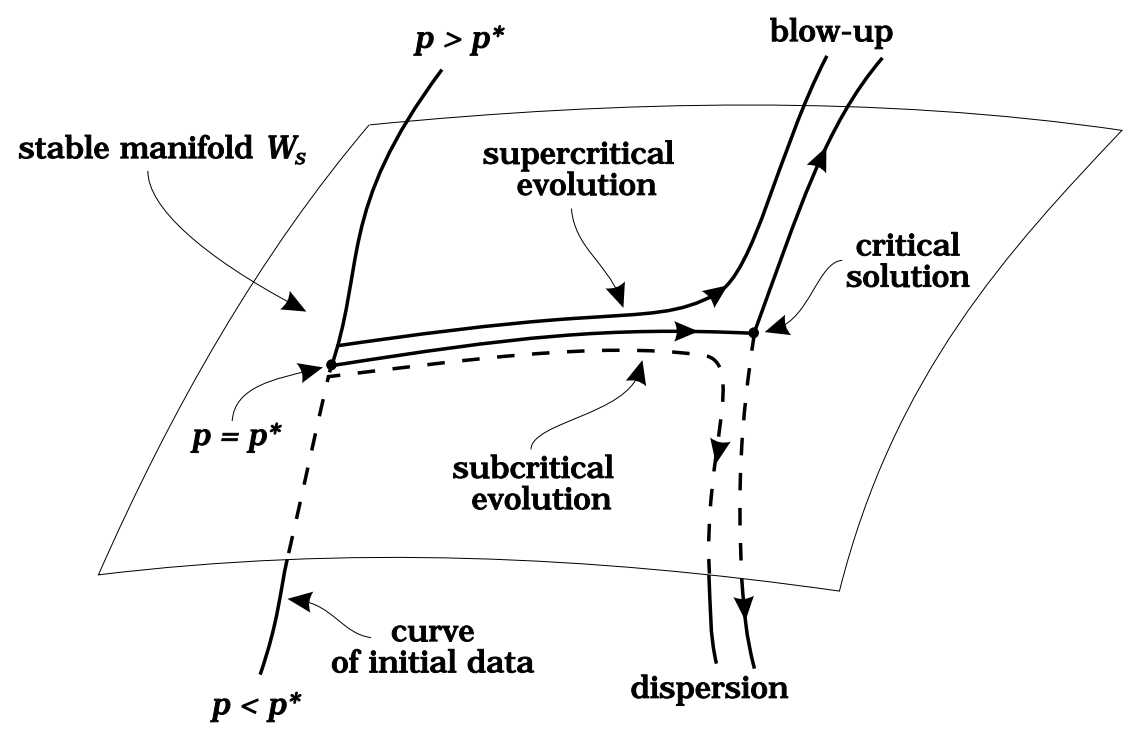

Figure. The conjectured phase space picture of the threshold behavior.

According to this picture, the threshold is given by the stable manifold $W_{S}\left(u^{*}\right)$ of a codimension one attractor $u^{*}$, hereafter called the critical solution. The critical data $\left\{\phi_{i}\left(p_{i}^{*}\right)\right\}$, corresponding to intersections of $W_{S}\left(u^{*}\right)$ with different interpolating one-parameter families of initial data, converge ${ }^{2}$ along $W_{S}\left(u^{*}\right)$ towards the critical solution. The marginally critical data, by continuity, initially remain close to $W_{S}\left(u^{*}\right)$ and approach $u^{*}$ for intermediate times but eventually are repelled from the vicinity of $u^{*}$ along its one-dimensional unstable manifold. Within this picture the universality of marginally critical dynamics in the intermediate asymptotics follows immediately from the fact that the same unstable

\footnotetext{
${ }^{1}$ Strangely enough, this general picture of the threshold behavior has been first established (as far as I know) in the context of arguably the most complicated nonlinear wave equations, namely Einstein's equations. This part of my presentation is in fact a model-independent synopsis of what has been first done by general relativists (see the example 3 and the review [8] for more details).

${ }^{2}$ Perhaps it is worth stressing that for the conservative wave equations considered here, the convergence to attractors (which is due to radiation of energy to infinity) is always meant in a local sense.
} 
mode dominates the evolution of all solutions. The nature of the critical solution itself depends on a model. Typically, $u^{*}$ is a static (that is time-independent) solution or a self-similar solution. In the remainder of this contribution, I discuss three models in which the threshold behavior has been recently analyzed.

\section{Wave equation with quintic nonlinearity}

Consider the nonlinear wave equation

$$
u_{t t}=\triangle u+u^{5}, \quad t>0, \quad x \in \mathbf{R}^{3}
$$

with spherically symmetric initial data $u(0, x)=f(r), u_{t}(0, x)=g(r)$. In this case it has long been known that: (i) solutions with small initial data exist globally and converge asymptotically to zero (this was first shown by John [9] for data of compact support and later extended by others for slowly decaying data), and (ii) large data solutions (in particular all data with negative energy) blow-up in finite time [11]. Equation (3) has the static spherically symmetric solution

$$
f(r)=\frac{1}{\sqrt{1+r^{2} / 3}}
$$

which, due to the scaling symmetry of (3), gives rise to a continuous family of solutions $f_{a}(r)=a f\left(a^{2} r\right)$. Substituting $u(t, x)=f(r)+w(t, x)$ into (3) and linearizing, we obtain the evolution equation for the perturbation $w$

$$
w_{t t}=(\triangle-V) w, \quad \text { where } \quad V=-\frac{5}{\left(1+r^{2} / 3\right)^{2}} .
$$

One can easily show that the potential $V(r)$ admits exactly one bound state which means that around $f$ there is exactly one growing mode $w=e^{\lambda t} \xi(r)(\lambda>0)$. Numerical computation yields $\lambda \approx 1.100167$. According to our preceding discussion, the solution $f$ is thus a candidate for the critical solution. To verify this, my student Nikodem Szpak [15] has investigated the nonlinear evolution of the growing mode and found that, depending on the sign of its amplitude, the solution either disperses or blows up in finite time. This demonstrated that, in fact, $f$ is the critical solution whose codimension one stable manifold plays the role of the threshold. The blowup manifests itself in the unbounded growth of the solution near $r=0$ at the rate $(T-t)^{-1 / 2}$ where $T$ is the blowup time. Note that $u=\left(\frac{3}{4}\right)^{1 / 4}(T-t)^{-1 / 2}$ is a singular stable solution of (3) so the blowup can be interpreted as (local) convergence to this solution. The evolution of marginally critical solutions in the intermediate asymptotics has the form

$$
u(t, r)=f_{a}(r)+c(p) e^{\lambda_{a} t} \xi_{a}(r)+\text { decaying modes }
$$

for some $a$, where $\lambda_{a}=a^{2} \lambda$ and $\xi_{a}(r)=\xi\left(a^{2} r\right)$. The small constant $c(p)$, which is the only vestige of initial data, quantifies the admixture of the unstable mode - for precisely critical data $c\left(p^{*}\right)=0$. The "lifetime" $T^{*}$ of the transient phase during which the linear approximation (6) is valid is determined by the time in which the unstable mode grows to a finite size, that is $c(p) e^{\lambda_{a} T^{*}} \sim O(1)$. Using $c(p) \approx c^{\prime}\left(p^{*}\right)\left(p-p^{*}\right)$, this gives $T^{*} \sim$ $-\frac{1}{\lambda_{a}} \ln \left|p-p^{*}\right|$. Thus, the larger $\lambda_{a}$, the better fine-tuning is required to see the solution $f_{a}$ clearly pronounced as the intermediate attractor. 
The rate of convergence of critical and marginally critical data to the critical solution is determined by the spectrum of decaying modes in (6). This spectrum can be obtained by solving (5) with the outgoing wave boundary condition at spatial infinity. Mathematically this is equivalent to finding resonances in the potential $V(r)$. Physically the damping is due to the loss of energy by radiation. It turns out that the least damped mode around $f_{a}$ is nonoscillatory (in quantum mechanics such a resonance is called an antibound state) and decays approximately as $e^{-0.08 a^{2} t}[15]$.

\section{Wave maps}

Let $M$ be a spacetime with a metric $\eta$ and $N$ be a Riemannian manifold with a metric $g$. The wave map $U: M \rightarrow N$ is defined as a critical point of the action

$$
S(U)=\frac{1}{2} \int_{M} g_{A B} \frac{\partial U^{A}}{\partial x^{a}} \frac{\partial U^{B}}{\partial x^{b}} \eta^{a b} \sqrt{-\eta} d^{4} x .
$$

The associated Euler-Lagrange equations

$$
\partial^{a} \partial_{a} U^{A}+\Gamma_{B C}^{A}(U) \partial_{a} U^{B} \partial^{a} U^{C}=0
$$

constitute the system of semilinear wave equations, where $\Gamma$ 's are the Christoffel symbols of the metric $g$. The interest in wave maps stems from the fact that they contain many features of more complex relativistic models but are much simpler to study. In particular, the investigation of questions of global existence and formation of singularities for wave maps can give insight into the analogous, but much more difficult, problems in general relativity.

Let us consider the case where $M=\mathbf{R}^{3+1}$, the $3+1$ dimensional Minkowski spacetime, and $N=S^{3}$, the unit 3-sphere. In polar coordinates on $\mathbf{R}^{3+1}$ and $S^{3}$, the respective metrics are $\eta=-d t^{2}+d r^{2}+r^{2} d \omega^{2}$ and $g=d u^{2}+\sin ^{2}(u) d \Omega^{2}$ where $d \omega^{2}$ and $d \Omega^{2}$ are the standard metrics on $S^{2}$, and $u \in[0, \pi]$. Assume the spherically symmetric ansatz $U(t, r, \omega)=(u(t, r), \Omega=\omega)$. Then, equation (8) reduces to

$$
u_{t t}=u_{r r}+\frac{2}{r} u_{r}-\frac{\sin (2 u)}{r^{2}} .
$$

The initial value problem for this equation has been studied by several authors with the result that: (i) solutions with small initial data exist globally in time [10, 14], and (ii there exist smooth initial data which lead to blowup in finite time. An example of (ii) is due to Shatah [13] who found the self-similar solution of (8), $f_{0}=2 \arctan \left(\frac{r}{T-t}\right)$. This solution is perfectly smooth for $t<T$ but it breaks down at $t=T$.

The formation of singularities and the threshold behavior for equation (9) have been recently studied numerically in [2]. The first outcome of these studies is that the solution $f_{0}$ is stable and possesses a large basin of attraction - this means that the self-similar blowup found by Shatah is in fact generic. The second outcome is the identification of the critical solution as another self-similar solution $f_{1}\left(\frac{r}{T-t}\right)$ which has one unstable mode (besides the gauge mode corresponding to the freedom of choosing the blowup time $T$ ). The existence of this solution was proved by a shooting technique [1]. In terms of the similarity variables $\rho=r /(T-t)$ and $\tau=-\ln (T-t)$, the evolution of marginally critical data in the intermediate asymptotics has formally the same form as (6)

$$
u(t, r)=f_{1}(\rho)+c(p) e^{\lambda \tau} \xi(\rho)+\text { decaying modes, }
$$


where $\lambda$ is the only unstable (that is positive) eigenvalue and $\xi(\rho)$ is the corresponding eigenfunction. Since in this case $\lambda \approx 6.33362$ is rather large, the duration of the intermediate asymptotics is relatively short even for very well tuned data.

\section{Einstein's equations}

Consider a spherical distribution of matter and let it collapse under its own weight. The dynamics of this process, modeled by Einstein's equations, can be understood in terms of the competition between the gravitational attraction and repulsive internal forces (due, for instance, to kinetic energy of matter or pressure). If the initial configuration is dilute, then the repulsive forces "win" and the collapsing matter will rebound or implode through the center, and eventually will disperse. On the other hand, if the density of matter is sufficiently large, some fraction of the initial mass will form a black hole. This kind of behavior has been established rigorously by Christodoulou in the case of gravitational collapse of a massless scalar field [6, 7]. Christodoulou's results clearly indicated the existence of the threshold for black hole formation but left open the key question: what is the mass of a black hole at the threshold? The program of studying this question numerically was carried out with remarkable success by Choptuik [4]. Using the basic technique of evolving families of initial data interpolating between no-black-hole and blackhole spacetimes, Choptuik found that:

(i) The masses of black holes formed from marginally supercritical data satisfy the scaling law $M_{b h} \sim\left(p-p^{*}\right)^{\gamma}$ where the critical exponent $\gamma \approx 0.37$ is universal. Thus by fine tuning the parameter $p$ (which physically measures the degree of concentration of mass) one can make an arbitrarily tiny black hole. Note that the strength of the gravitational field at the horizon of a black hole is inversely proportional to the black hole mass, thus the threshold behavior is a par excellence strong-field phenomenon.

(ii) The critical solution is a limiting, sort of zero mass black hole ${ }^{3}$. This solution was found to possess a rare symmetry of discrete self-similarity, that is

$$
u^{*}(r, T-t)=u^{*}\left(r / e^{\Delta},(T-t) / e^{\Delta}\right),
$$

where the singularity formation time $T$ is family dependent but the discrete self-similarity scale $\Delta \approx 3.44$ is universal. The discrete self-similarity of the critical solution is widely considered as the most intriguing aspect of the threshold phenomena in gravitational collapse.

Seven years and more than one hundred papers after Choptuik's discovery, we know that the threshold behavior in many other models of gravitational collapse is qualitatively the same as for the scalar field although is some cases the critical solution is continuously (rather than discretely) self-similar. Moreover, if a model admits a stationary solution with a single instability, then such a solution can play the role of a critical solution [5, 3]. In this case black hole formation turns on with finite mass. In the literature the threshold behavior in gravitational collapse is referred to as the type II or type I behavior, depending on whether a critical solution is self-similar or stationary. This terminology emphasizes the formal analogy of threshold behavior with second and first order phase transitions in

\footnotetext{
${ }^{3}$ This result made it to a front page story in the New York Times [12] covering the concession of a bet by Steven Hawking on the validity of cosmic censorship conjecture. According to this conjecture, the singularities produced in the gravitational collapse must (generically) be hidden in a black hole so that no "naked" singularities can occur. Choptuik's critical solution is an example of a naked singularity, however, being nongeneric, it is not a counterexample to cosmic censorship, contrary to some claims in the NYT.
} 
statistical physics. The examples 1 and 2 discussed above in a sense can be considered as toy-models of type I and II behaviors, respectively — actually the analysis of these examples was motivated by attempts to get an analytic insight into some aspects of critical gravitational collapse. It would be most interesting to have a toy-model exhibiting discrete self-similarity. The work towards constructing such a model is in progress.

The computer simulations were instrumental in the discovery and understanding of the threshold phenomena, so this brief note would not be complete without a few sentences about numerical methods. All the evolution equations discussed above were solved by finite difference techniques. Since the singularity formation involves the development of structure on a vanishingly small scale, fixed grid schemes are inadequate. To capture the structure of the developing singularity, it is necessary to use an adaptive method which refines the spatio-temporal grid near the singularity. For example, in the case of wave maps, unless the time steps are sufficiently reduced as $t \rightarrow T$, the scheme steps over the blowup time and the singularity formation is missed completely. But even with mesh refinement, it is hard to assess if the produced singularities are not numerical artifacts unless one has some a priori analytical insight into a problem. It should be stressed that the accurate and reliable simulation of singularity formation is very difficult by its nature and only the most careful confrontation of outputs generated by different methods (in the ideal case combined with analytical information) can make one feel confident about the results of simulations.

\section{Acknowledgements}

This research was supported in part by the KBN grant 2 P03B 01016.

\section{References}

[1] Bizoń P, Equivariant Self-Similar Wave Maps from Minkowski Spacetime into 3-Sphere, Comm. Math. Phys., 2000, V.215, 45-56.

[2] Bizoń P, Chmaj T and Tabor Z, Dispersion and Collapse of Wave Maps, Nonlinearity, 2000, V.13, 1411-1423.

[3] Bizoń P and Chmaj T, Formation and Critical Collapse of Skyrmions, Phys. Rev., 1998, V.58, 041501.

[4] Choptuik M W, Universality and Scaling in Gravitational Collapse of a Massless Scalar Field, Phys. Rev. Lett., 1993, V.70, 9-12.

[5] Choptuik M W, Chmaj T and Bizoń P, Critical Behavior in Gravitational Collapse of a Yang-Mills Field, Phys. Rev. Lett., 1996, V.77, 424-427.

[6] Christodoulou D, The Formation of Black Holes and Singularities in Spherically Symmetric Gravitational Collapse, Comm. Pure Appl. Math., 1991, V.44, 339-373.

[7] Christodoulou D, Bounded Variation Solutions of the Spherically Symmetric Einstein-Scalar Field Equations, Comm. Pure Appl. Math., 1993, V.46, 1131-1220.

[8] Gundlach C, Critical Phenomena in Gravitational Collapse, Adv. Theor. Math. Phys., 1998, V.2, 1-50.

[9] John F, Blow up of Solutions of Nonlinear Wave Equations in Three Dimensions, Manuscripta Math., 1997, V.28, 235-268.

[10] Kovalyov M, Long-Time Behaviour of Solutions of a System of Nonlinear Equations, Comm. PDE, 1987, V.12, 471-501. 
[11] Levine H A, Instability and Nonexistence of Global Solutions to Nonlinear Wave Equations, Trans. Amer. Math. Soc., 1974, V.192, 1-21.

[12] A Bet on a Cosmic Scale, and a Concession, Sort of, New York Times, February 12, 1997, Section 1, P.1.

[13] Shatah J, Weak Solutions and Development of Singularities of the SU(2) $\sigma$-Model, Comm. Pure Appl. Math., 1988, V.41, 459-469.

[14] Sideris T, Global Existence of Harmonic Maps in Minkowski Space, Comm. Pure Appl. Math., 1989, V.42, 1-13.

[15] Szpak N, Universal Dynamics at the Threshold for Nonlinear Wave Equations, Master's Thesis (in Polish), Jagellonian University, 1999. 